\title{
Symmetric Ventral Brainstem Lesion in Leptomeningeal Carcinomatosis
}

\author{
Kengo Maeda ${ }^{1}$, Hiroshi Wada ${ }^{2}$, Yoshitomo Ozaki ${ }^{3}$ and Akitoshi Inoue ${ }^{4}$
}

Key words: leptomeningeal carcinomatosis, lung cancer, adenocarcinoma, MRI, brainstem

(Intern Med 58: 759-760, 2019)

(DOI: 10.2169/internalmedicine.1469-18)

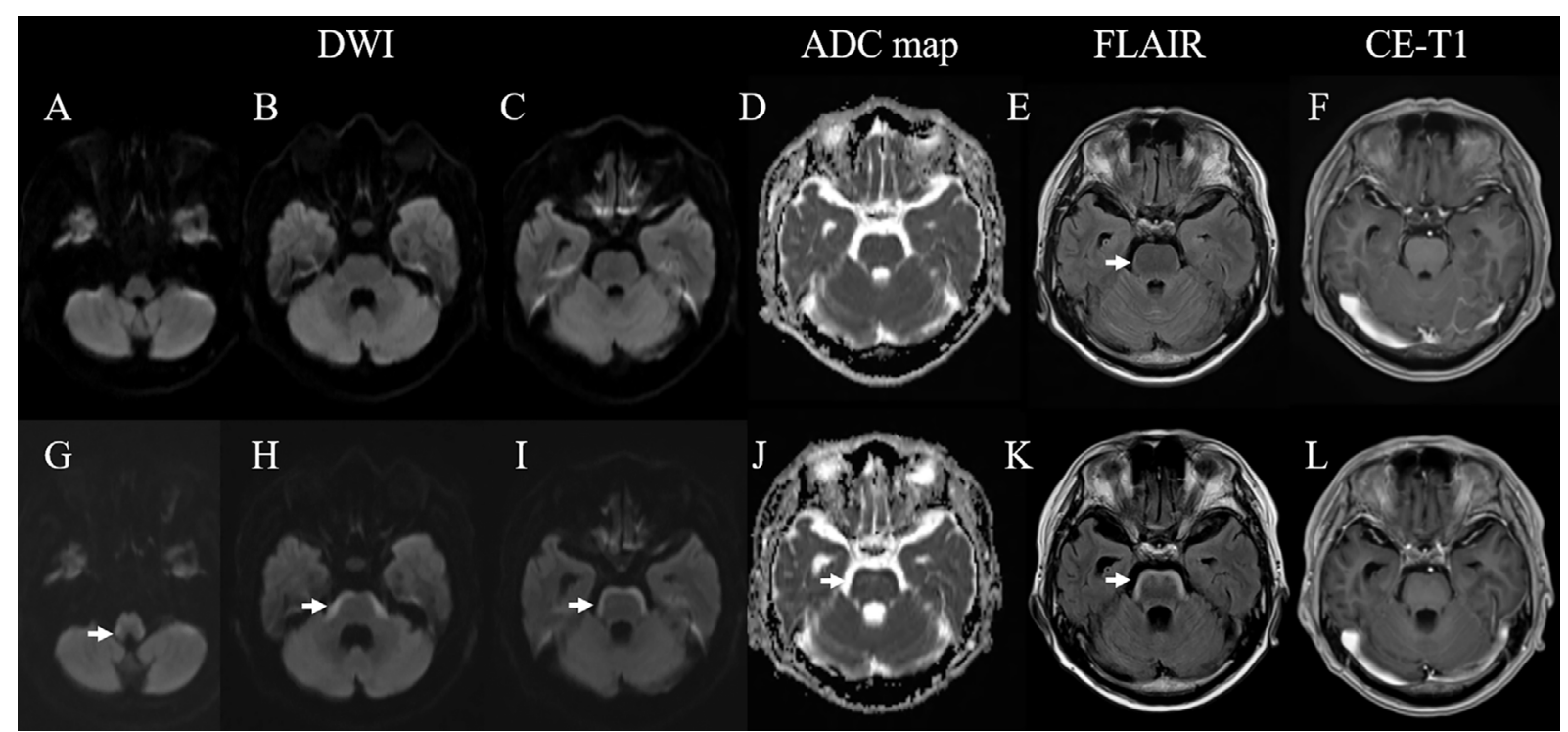

Picture 1.

Recently, novel magnetic resonance imaging (MRI) findings in leptomeningeal carcinomatosis due to lung adenocarcinoma have been reported $(1,2)$. Case 1 was a 55 -year-old man presenting with dizziness and vomiting. He had been diagnosed with lung adenocarcinoma carrying an exon 19 deletion of the epidermal growth factor receptor gene. Malignant cells were found in his cerebrospinal fluid (CSF). Findings on MRI diffusion-weighted imaging (DWI, Picture 1A-C), apparent diffusion coefficient (ADC) mapping (Picture 1D), and contrast-enhanced T1-weighted imaging (CE-T1, Picture 1F) were not remarkable. Fluid attenuating inversion recovery imaging (FLAIR) without the administration of contrast-media revealed a symmetric hyperintense le- sion of the ventral surface of the pons (Picture 1E, arrow). MRI after four months (Picture 1G-L) showed the spread of this lesion to the medulla oblongata (Picture 1G, arrow) and hyperintensity on DWI (Picture 1G-I, arrows) and hypointensity on ADC mapping (Picture 1J, arrow). Case 2 was a 77-year-old man with lung adenocarcinoma showing trochlear nerve palsy. Malignant cells were also observed in his CSF. His MRI findings (Picture 2) were similar to those in Case 1. Although it is hypothesized that malignant cells infiltrate into the perforating arteries along the brainstem surface and cause microinfarctions (2), there is no pathological evidence supporting this notion.

${ }^{1}$ Department of Neurology, National Hospital Organization Higashi-ohmi General Medical Center, Japan, ${ }^{2}$ Department of Respiratory Medicine, National Hospital Organization Higashi-ohmi General Medical Center, Japan, ${ }^{3}$ Department of General Thoracic Surgery, National Hospital Organization Higashi-ohmi General Medical Center, Japan and ${ }^{4}$ Department of Radiology, National Hospital Organization Higashi-ohmi General Medical Center, Japan

Received: May 7, 2018; Accepted: August 29, 2018; Advance Publication by J-STAGE: November 19, 2018

Correspondence to Dr. Kengo Maeda, maeda-kengo@shiga-hosp.jp 


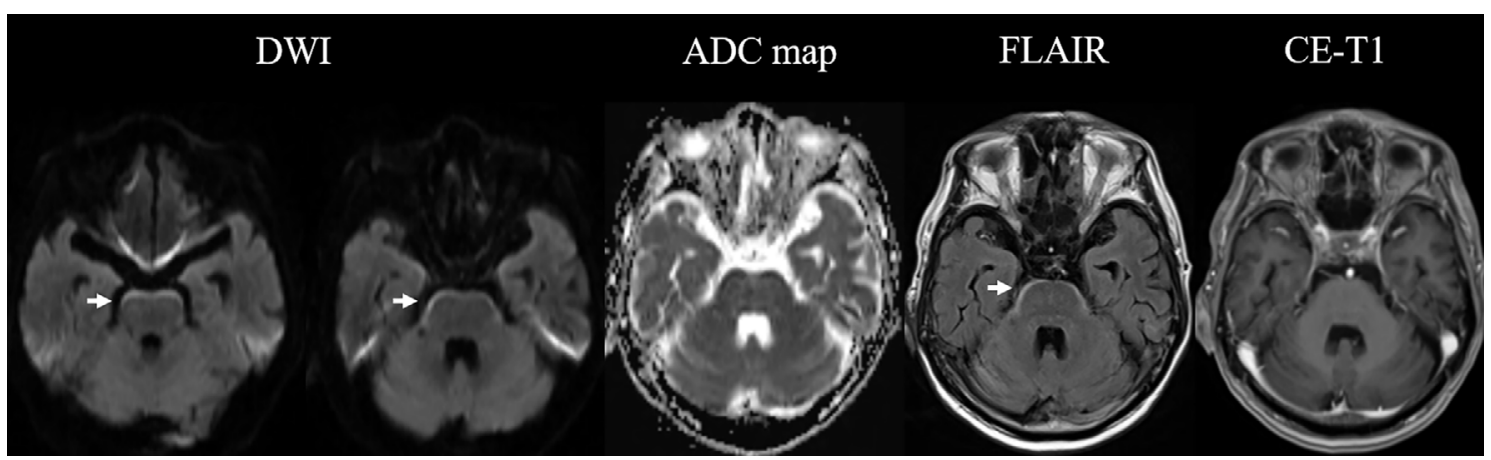

Picture 2.

The authors state that they have no Conflict of Interest (COI).

\section{References}

1. Khil EK, Lee AL, Chang K-H, Yun TJ, Hong HS. Symmetrical curvilinear cytotoxic edema along the surface on the brain stem: a probable new magnetic resonance imaging finding of leptomeningeal carcinomatosis. Medicine 94: e1053, 2015.
2. Cheng C-Y, Hsu C-Y, Tsai Y-H, et al. Novel anterior brainstem magnetic resonance imaging findings in non-small cell lung cancer with leptomeningeal carcinomatosis. Front Neurol 8: 579, 2017.

The Internal Medicine is an Open Access journal distributed under the Creative Commons Attribution-NonCommercial-NoDerivatives 4.0 International License. To view the details of this license, please visit (https://creativecommons.org/licenses/ by-nc-nd/4.0/).

(C) 2019 The Japanese Society of Internal Medicine Intern Med 58: 759-760, 2019 